\title{
Surfaces
}

\section{COLLOQUE QUEER SITES (MAI 1993). L'ÉTAT DU JEU : QUELQUES RUBRIQUES DE LA THÉORIE QUEER}

\section{Christophe Park}

Volume 3, 1993

URI : https://id.erudit.org/iderudit/1065115ar

DOI : https://doi.org/10.7202/1065115ar

Aller au sommaire du numéro

Éditeur(s)

Les Presses de l’Université de Montréal

ISSN

1188-2492 (imprimé)

1200-5320 (numérique)

Découvrir la revue

Citer ce compte rendu

Park, C. (1993). Compte rendu de [COLLOQUE QUEER SITES (MAI 1993). L’ÉTAT DU JEU : QUELQUES RUBRIQUES DE LA THÉORIE QUEER]. Surfaces, 3.

https://doi.org/10.7202/1065115ar
Résumé de l'article

En mai 1993 s'est tenu, au New College de l'Université de Toronto, un colloque d'études culturelles lesbiennes et gaies intitulé Queer Sites: Bodies at Work, Bodies at Play. Reprenant quelques-uns des thèmes abordés au cours de cet événement, l'auteur fait état des défis pragmatiques et épistémologiques auxquels sont actuellement confrontées les études lesbiennes et gaies.
Copyright @ Christophe Park, 1993

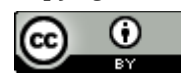

Ce document est protégé par la loi sur le droit d'auteur. L’utilisation des services d'Érudit (y compris la reproduction) est assujettie à sa politique d'utilisation que vous pouvez consulter en ligne.

https://apropos.erudit.org/fr/usagers/politique-dutilisation/ 


\title{
COMPTE-RENDU DE COLLOQUE
}

\author{
QUEER SITES (MAI 1993)
}

L'éTAT DU JEU:

QUELQUES RUBRIQUES DE LA THéORIE QUEER

Christophe Park

\section{RÉSUMÉ}

En mai 1993 s'est tenu, au New College de l'Université de Toronto, un colloque d'études culturelles lesbiennes et gaies intitulé Queer Sites: Bodies at Work, Bodies at Play. Reprenant quelques uns des thèmes abordés au cours de cet événement, l'auteur fait état des défis pragmatiques et épistémologiques auxquels sont actuellement confrontées les études lesbiennes et gaies.

\section{ABSTRACT}

In may 1993, Toronto University's New College hosted a conference in lesbian and gay cultural studies entitled Queer Sites: Bodies at Work, Bodies at Play. Taking up some of the issues brought up by the participants, the author exposes the pragmatic and epistemological challenges lesbian and gay studies must face. 
Parler de théorie lesbienne et gaie à un auditoire universitaire euroaméricain contemporain, c'est nécessairement attiser la controverse; d'abord, bien sûr, au sein de l'auditoire - -- dont les réactions s'échelonneront le long d'un spectre allant de l'intolérance envers l'étalage des détails de la vie privée, à l'impatience face au rabâchage lassant de revendications d'évidences -- mais aussi chez celles et ceux qui articulent le discours théorique. En effet, puisque le désir pour les individus du même sexe est au fondement de la discipline des études lesbiennes et gaies, les théoriciens sont plus conscients que quiconque des implications de l'évocation de ce désir, à la fois marginal et central, aussi bien que secret -le plus souvent de Polichinelle. Et, à travers les considérations théoriques qui leur donnent accès à la discipline, ils sont embarrassés plus que quiconque par la formulation de vérités qui, à plusieurs niveaux, sont des évidences grossières, souvent tout juste susceptibles d'entretenir l'hostilité froide d'un statu quo composé d'indifférence polie.

Le projet demeure, cependant. Depuis bientôt cinq ans, dans plusieurs universités anglo-américaines[1], il fonde -- à l'encontre d'un conservatisme institutionnel dont les restrictions les plus apparentes n'ont sans doute pas besoin d'être identifiées ici -- des départements et des programmes d'études lesbiennes et gaies. Il nourrit aussi une réflexion écrite, qui a déjà jeté les bases d'un corpus, et une activité de congrès intellectuel fort riche dont j'ai moi-même été témoin, au mois de mai de cette année à Toronto, lors du colloque Queer Sites. Si tant de volontés et d'esprits se sont réunis, bravant non seulement les disparités de la différence sexuelle, mais aussi les difficultés procédant de la structuration ouverte/publique du privé/secret de leur préférence sexuelle, le locuteur que voici ne devrait-il pas, à son tour, avoir le courage de s'imprégner de la même énergie anti-conservatrice, pour s'opposer à la stase polie de tous les statu quo et véhiculer, à son tour, certaines évidences? "It's only by being shameless about risking the obvious that we happen into the vicinity of the transformative" (Sedgewick 1990, 22).

L'une des notions les plus productives qui dérive des propositions de refonte ou de réforme canoniques en théorie lesbienne et gaie est, somme toute, analogue au projet qui sous-tend la relecture et la ré-anthologisation féministe du canon littéraire. Il s'agit de mettre en lumière un nombre de lectures et de questionnements spécifiques "perhaps new, available in a heuristically powerful, productive, and significant form for other readers to perform on literary and social texts with, ideally, other results" (Sedgewick 14). Cette puissance heuristique est, comme on l'imagine, fortement déterminée par la volonté de s'opposer à l'homophobie institutionnelle. Il faut reconnaître que les études féministes ont materné, fut-ce parfois à bout de bras, les études lesbiennes et gaies. Toutefois, l'heuristique du projet 
queer étant déterminée par une pratique sexuelle -- et caractérisée, ajouterait-on, par les enjeux de la différence sexuelle -- il faut aussi comprendre que si la mère et l'enfant posent des questions analogues, il ne saurait y avoir congruence au niveau des théories qu'engendrent ces questionnements.

Modelées sur les débats de la modernité euro-américaine, les études lesbiennes et gaies, dans le post-Stonewall[2] universitaire américain en particulier, en ont reproduit les schèmes discursifs avec fidélité. Entre la fin des années soixante et la fin des années quatre-vingt, point de réflexion en sciences humaines sur les enjeux gais et lesbiens qui n'engage d'une façon ou d'une autre, sous l'avatar d'une homosexualité construite contre l'homosexualité d'essence, le débat difficile à taire entre nature et culture. C'est sans doute le travail de John Boswell, Christianity, Social Tolerance, and Homosexuality: Gay People in Western Europe from the Beginning of the Christian Era to the Fourteenth Century (Chicago: 1980) qui formule le plus clairement l'argument d'une prédisposition au désir pour les individus du même sexe qui transcende les conceptions culturelles de la sexualité et qui permet de parler d'une essence homosexuelle. Il y a un décalage épistémologique remarquable entre cette première phase des études lesbiennes et gaies, où la théorie essentialiste se mesure au constructivisme foucauldien -- pour lequel la catégorie d'une sexualité homosexuelle n'a de sens que dans un nombre limité de cultures, dont l'Occident moderne -- et la deuxième phase, dite queer, qui semblerait chercher non pas une loyauté à l'un ou l'autre terme de la dyade nature/culture, mais bien une alternative à son obsolescence.

La crainte qu'exprime Sedgewick à ce sujet peut sembler procéder de l'évidence, voire de la paranoïa, mais elle est riche d'implications pour une théorie queer qui voudrait aller plus loin: "my fear is that there currently exists no framework in which to ask about the origins or development of individual gay identity that is not already structured by an implicit, transindividual Western project or fantasy of eradicating that identity." (Sedgewick 41). La critique d'une homophobie institutionnelle -- il s'agit ici de propos rapportés d'un jugement de la Cour suprême américaine -- où la tentative de défendre la pratique de la sodomie en évoquant les libertés individuelles tiendrait au mieux de la facétie, ne peut que faire réfléchir sur la façon dont les puissants, précisément parce qu'ils sont obtus, viennent justement mieux à bout de leurs ennemis: "The power of our enemies over us is implicated, not in their command of knowledge, but precisely in their ignorance" (Sedgewick 7). Si l'on perçoit des relents de la théorie de la conspiration dans cette affirmation, il faut se rappeler que le véritable danger ne réside pas tant dans les réseaux homophobes de l'institution -- qui cherchent par des questions d'ontogénie à désamorcer le pétard désagréable de la sexualité gaie et lesbienne quand celle-ci intervient au niveau des analyses -- que dans le travail délicat et dangereux de l'analyse gaie/lesbienne qui s'engage souvent sans préparation adéquate dans sa version du débat nature/culture; encore une crainte exprimée par Sedgewick: "I fear that the special volatility of postmodern bodily and 
technological relations may make such an attempt peculiarly liable to tragic misfire" (Sedgewick 1990, 41).

C'est-à-dire, en termes simplistes, que l'enthousiasme et les bonnes intentions ne suffisent pas à faire avancer le projet. Bien des réflexions, sans doute homo-positives, se sont avérées rétrogrades dans leur façon de mettre en jeu le fonctionnement subtil des intérêts institutionnels. On pense notamment ici au type d'argumentation qui s'appuie sur la soi-disant "nonpertinence" d'une sexualité lesbienne ou gaie dans l'exercice de la théorie littéraire ou culturelle -- le "placard" auquel se réfère le titre de l'ouvrage d'Eve Kosofsky Sedgewick, Epistemology of the Closet. Le placard n'est pas toujours un sombre réduit d'oppression infame et de honte secrète; il est possible, l'opération est même fréquente, de lire l'homosexualité dans le canon littéraire dans des termes qui s'échelonnent le long d'un spectre allant du prestige et du magnétisme à la défiance et à l'aliénation du soi, en passant par la vulnérabilité et la sensiblerie! Il me semble qu'on exprime par ces termes une nostalgie du placard comme espace qui permet de ranger convenablement les désordres sexuels, loin des yeux, loin de nos préoccupations critiques, "non-pertinents" en fin de compte.

Les stratégies d'enquête des études gaies et lesbiennes, qui ne peuvent qu'être informées par les questions de différence sexuelle, sont, du fait même de la floraison récente (à la fois abondante et fragile) de la discipline, souvent menées à des choix tenant plus de la loyauté à une tradition établie que de l'entreprise pionnière et téméraire. Les études queer, à l'encontre justement d'une tradition établie de lesbianisme séparatiste, isolant l'analyse féministe des termes de la recherche homo-masculine, choisit de risquer, pour une durée indéterminée, la mise en présence des deux stratégies. L'option de les forclore l'une de l'autre dérive bien sûr de l'heuristique anti-homophobe qui motive l'avant-garde queer: un différé, sans limite précise de durée, qui refuse de rendre compte à l'une ou l'autre partie et qui, en cela, exerce le refus terminologique et rhétorique qui définit toute avant-garde. Ce différé met l'emphase sur l'acceptation nécessaire, dans un projet d'analyse queer, d'une vision homo-masculine, "a gay male-oriented analysis that would have its own claims to make for an illuminating centrality" (Sedgewick 16)

Le projet queer, la validation en fait de son heuristique anti-homophobe, vise sans doute à refocaliser bien des enjeux communs aux lesbiennes, aux gais, aux femmes et aux hommes, où la sexualité peut n'être pas toujours éclatée à l'infini de la différence sexuelle: "the fostering of our ability to arrive at understandings of sexuality that will respect a certain irreductibility in it to the terms and relations of gender" (Sedgewick 16). Ce qui anime les études queer, du moins ce sur quoi insistent les ouvrages les plus représentatifs de son corpus relativement mince, n'est pas moins qu'un refus de la binarité conventionnelle qui met en oppostition l'hétérosexualité et l'homosexualité et qui peut être rendue par la métaphore du placard -- dans lequel on ne 
peut jamais être définitivement enfermé mais dont on ne peut pas non plus sortir tout-à-fait. C'est sur ce difficile paradoxe de l'intérieur et de l'extérieur que doit travailler le projet queer. Par-delà le débat de l'exclusion -- ou nature ou culture -- se trouve la nécessité de repenser les termes du dehors et du dedans, "to recognize that any outside is formulated as a consequence of a lack internal to the system it supplements. The greater the lack on the inside, the greater the need for an outside to contain and to defuse it, for without that outside, the lack on the inside would become all too visible" (Fuss 3).

Il est à noter que le rapport entre une avant-garde queer, dont j'ai fait mention plus haut, et une avant-garde radicale universitaire est beaucoup plus complexe que les apparences de sympathie, voire de collusion, qui le traversent ne le laisseraient croire. Les éléments queer dans les marges du champ intellectuel, plus précisément universitaire, ne peuvent finalement s'envisager comme étant séparés, en dehors, de la zone centrale de pouvoir -- d'appui, d'intérêt, de soutien -- par ceux qui forment la zone-tampon et qu'on pourrait qualifier méchamment de fashion victims de la mode queer. A partir du moment où l'on se campe sur les marges, à l'extérieur, la position polémique qu'on assume voile la véritable collusion entre les institutions et les avant-gardes: "Any misplaced nostalgia for or romanticization of the outside as a privileged site of radicality immediately gives us away, for in order to idealize the outside we must already be, to some degree, comfortably entrenched on the inside" (Fuss 5). Ceux qui sont en procès d'institutionnalisation, mais dont les affinités pour l'espace liminal où se situent les études queer sont gouvernées par l'habitus acquis lorsque les intervenants de ce champ intermédiaire étaient identifiés comme marge extrême par l'institution, ne sont donc pas tant à cibler dans l'heuristique du projet queer que les obstacles, internes et externes, à l'élaboration d'une théorie des frontières de la sexualité -- qui nous aiderait à mettre nos corps à l'oeuvre dans les nouvelles dispositions occasionnées par le désir et le plaisir qui sont en jeu dans le champ culturel.

Je ferai part ici de quelques éléments du discours-programme du colloque, prononcé par Elizabeth Grosz, de l'Université Monash, et intitulé "Experimental Desire: Bodies and Pleasure in Queer Theory" ("L'expériencedésir: corps et plaisir en théorie queer"). Ce texte illustre bien la double difficulté d'exprimer la liminalité nécessaire à une théorie queer, et d'accepter ce que Teresa de Lauretis envisage comme "an agency of social process whose mode of functioning is both interactive and yet resistant, both participatory and yet distinct, claiming at once equality and difference" (de Lauretis iii). Le propos de la conférencière s'est d'abord ouvert sur deux remarques visant à modifier le titre de sa prestation. La première, d'une plaisante ironie, invoquait comme contexte pour la communication l'égide de la liberté universitaire qui, pour toute réflexion théorique, s'est avérée le plus souvent être un "pilier de stagnation". L'auditoire était prévenu, la barre allait être tenue dans la houle de la controverse; et ce qui allait suivre risquait d'offenser certains partisans des diverses causes correctes venues revendiquer une place au colloque. La deuxième remarque permettait à 
Grosz de se situer, en tant que femme et en tant qu'intellectuelle, par rapport à l'orientation théorique qui allait dominer sa communication. Son évocation de la "fascination et de l'ambivalence" qui déterminent le rapport des femmes à la psychanalyse, ainsi que des nuances particulières de la définition psychanalytique de la perversité, laissait présager son choix subséquent d'élaborer le thème du/des corps et du plaisir -- qu'elle situe dans la zone ambiguè entre la pragmatique du désir et l'ontologie des corps -- en s'inspirant de la théorie psychanalytique contemporaine et, plus spécifiquement, des travaux de Deleuze.

Constatant que le propre de l'homophobie est de réduire le désir pour l'individu du même sexe à une ontologie, Grosz est ensuite amenée à affirmer avec insistance que la catégorie du queer se doit d'assumer, à l'encontre des coercitions des "communautés" gaies/lesbiennes, une posture défiante et toujours en mutation envers les modèles épistémologiques qu'elle conteste, ou qu'elle problématise. C'est donc là l'appel que fait Grosz, non seulement en se basant sur l'opposition deuleuzienne entre l'ontologie et la pragmatique, mais aussi en évoquant l'exemple des différences performatives irréfutables entre le sado-masochime lesbien et sa contrepartie homo-masculine. Cette considération de la spécificité du corps sexué n'a pas manqué de troubler une partie de l'auditoire. La réaction ne s'est toutefois manifestée que plus tard, au moment où Grosz fut soumise par ses interlocuteurs à une interrogation en règle, qui n'était pas sans rappeller l'homophobie institutionnelle dans sa façon de reclamer la mise en place de frontières fixes dans le champ de la sexualité.

Les notions d'action et de réaction, de relâchement et de restriction, évoquées plus tôt dans l'analyse deleuzienne du devenir des corps, furent reprises par Grosz pour tenter de répondre aux questions soulevées. Si l'on pose, au nom de la différence sexuelle, que le queer est un procès en progression et en expansion, à l'encontre des forces de l'institution homophobe qui, elles, sont en réaction tout aussi permanente, on se leurre gravement en assumant qu'il y a équation absolue entre discours et expérience. Reconnaître les limites de la sexualité est nécessaire, tout d'abord à l'économie personnelle en jeu dans l'action queer, et ensuite à la réaction anti-homophobe qui la caractérise. Cette valorisation des deux termes de la dyade action-réaction, plutôt qu'une sanction de la contestation réactive à sens unique, rappelle l'insistance du corpus queer sur le besoin de repenser la sortie et l'entrée, l'interne et l'externe, au nom d'une pragmatique qui saura contrer aux deux paliers les ontologies biaisées. S'il y a une pragmatique de femmes et d'hommes queer, il y aura bien entendu un être derrière les faits; l'important est de refuser la sédimentation en mettant à jour l'extase et l'oubli de soi dont est saturé le désir. La véritable subversion est celle de la production du plaisir pour le propre du plaisir.

Il serait futile de détailler ici les différentes orientations des communications offertes au cours du colloque, ou de tenter de rendre compte des chaudes 
discussions qu'ont occasionnées la plupart des exposés. Au cours de trois journées consacrées respectivement aux thèmes Homoacademica, Querying the Queer et Sex at the Boundaries, cent trente-huit conférencières et conférenciers se sont relayés dans un colloque qui, par son emphase sur l'orientation "lettres" des sciences humaines, s'est distingué de sa contrepartie québecoise, La ville en rose, qui s'est tenue à l'UQAM et à l'Université Concordia l'automne dernier. Les quelques interventions issues du domaine des sciences sociales et éducatives et de celui des arts ont pourtant permis de constater l'intérêt que portent ces autres disciplines à la constante redéfinition d'un placard de plus en plus transformé et méconnaissable.

Parmi les communications les plus marquantes, il faut souligner "Girls and Girls, Girls and Horses: Riding Positions (of Desire) in Queer Theory" d'Elspeth Probyn (Université de Montréal), une réflexion sur l'imprévisible comme principe d'individuation plutôt que comme fondement de l'individu, appliqué à l'image apparemment monologique des jeunes filles à cheval. Egalement: "Espaces féministes et espaces queer," de Jeannelle Savona (Université de Toronto), qui a su identifier les négligences et les exclusions du projet queer en les mesurant à l'expérience mûrie de la réflexion et de la pratique féministes. Enfin, l'intervention impromptue du ciné-vidéaste torontois Richard Fung, dont l'oeuvre est vouée à la prévention du VIH-sida. Fung a soulevé la question de la spécificité ethnique et de son articulation avec les problèmes de la différence sexuelle: l'abusive spécification de la différence étant souvent pour lui le symptôme d'une peur des relations interraciales.

\title{
Christophe Park
}

Département de Littérature comparée

\author{
Université de Montréal
}

Surface Page d'Acceuil/Home Page

\section{Bibliographie}


Abelove, Henry (et al., éd.). The Lesbian and Gay Studies Reader. London: Routledge, 1993.

Butler, Judith. Gender Trouble: Feminism and the Subversion of Identity. London: Routledge, 1990.

Doty, Alexander. Making Things Perfectly Queer: Interpreting Mass Culture. Minneapolis: University of Minnesota Press, 1993.

Fuss, Diana (éd.). Inside/Out: Lesbian Theories, Gay Theories. London: Routledge, 1991.

de Lauretis, Teresa. "Queer Theory: Lesbian and Gay Sexualities, An Introduction," In differences 3; 2 (1991).

Sedgewick, Eve Kosofsky. Epistemology of the Closet. Berkeley: University of California Press, 1990.

[1]Le grief d'anglo-centrisme formulé, à plusieurs reprises, contre le projet d'études lesbiennes et gaies/ queer est, dans une large mesure justifié. Sans pour autant soulever ici de fort pertinentes questions d'extensibilité vers le non-anglophone (ou le non-nanti, non-blanc, non-occidental, non-etc...) de l'espace discursif queer, rappelons qu'il existe dans les curriculums universitaires néerlandais et allemands (et dans la périphérie immédiate de certaines institutions universitaires italiennes) un intérêt spécifique pour les études gaies et lesbiennes qui s'articule autant sur des traditions et des historiques locaux de la discipline que sur le mimétisme idéologique de l'Amérique du Nord.

[2]Le terme Stonewall, dans le lexique gai et lesbien, se rapporte à la série de manifestations s'opposant, au cours de l'été 1969 à New York, aux descentes policières sur le Stonewall Inn, un bar gai dont la clientèle comprenait de nombreux travelos. Stonewall est un peu la ligne de partage des eaux entre la grande noirceure et la révolution bruyante que d'aucuns voudraient voir soldée avec l'avènement du sida. 